\title{
ON THE DIOPHANTINE EQUATION
}

$$
11+2^{x+2}+(7) 3^{y}=z^{2}
$$

\section{IDIR SADANI}

ABSTRACT. In this note, we investigate solutions of the Diophantine equation

$$
11+2^{x+2}+(7) 3^{y}=z^{2} \quad(x, y, z) \in \mathbb{N}^{3} .
$$

\section{Introduction}

In 1844 , C a t a la n in [2] conjectured that $(a, b, x, y)=(3,2,2,3)$ is a unique solution for the exponential Diophantine equation

$$
a^{x}-b^{y}=1 \quad(a, b, x, y) \in \mathbb{N}^{4} \quad \text { and } \quad \min (a, b, x, y)>1 .
$$

The proof of this conjecture is due to $\mathrm{Mih}$ a i les c u [3, 2004]. In 2011, S u v a r n a mani [1] found all non-negative integer solutions of diophantine equation

$$
2^{x}+p^{y}=z^{2}
$$

In 2012, C h o t c h a is t h it [4, using this theorem, found all solutions for the Diophantine equation $4^{x}+p^{y}=z^{2}$. In 2014, Y a h u i Y u and X i a ox u e L i 5] investigated solutions of equations of type

$$
2^{x}+b^{y}=c^{z}, \quad x, y, z \in \mathbb{N},
$$

where $b$ and $c$ are fixed coprime odd positive integers with $\min (b, c)>1$.

In this note, we give a proof of the following result.

\section{Main theorem}

Theorem 2.1. The Diophantine equation $11+2^{x+2}+(7) 3^{y}=z^{2}$ has a unique solution given by $(x, y, z)=(3,1,8)$.

(C) 2017 Mathematical Institute, Slovak Academy of Sciences. 2010 Mathematics Subject Classification: 11D61.

Keywords: exponential Diophantine equations. 
P r o o f. Let $x, y$ be non-negative integers and $z$ be an even non-negative integer such that

$$
11+2^{x+2}+(7) 3^{y}=z^{2}
$$

We need to consider several cases:

Case 1. If $y=0$. Then, $2^{x+2}+18=z^{2}$. If we take $z=2 k$, we obtain $2^{x+1}=$ $2 k^{2}-9$. But $2^{x+1}=0(\bmod 2)$. This is a contradiction.

Case 2. If $y=1$. Then, the equation becomes $2^{x+2}+2^{5}=z^{2}$. The solution of this equation is $(x, z)=(3,8)$ (see [1]). Thus, the triplet $(3,1,8)$ is a solution of our equation.

Case 3. If $y>1$. We have $11+2^{x+2}+(7) 3^{y}=4\left(1+2^{x}\right)+7\left(1+3^{y}\right)=z^{2}$. Since $z$ is even, then, $y$ is odd because $7\left(1+3^{2 l+1}\right)=0(\bmod 4)$. Next, we can write $4\left(1+2^{x}\right)+7\left(1+3^{y}\right)$ as $2^{j} c+2^{j^{\prime}} c^{\prime}$. Then, we deduce that $j=2$ and $1+3^{y}=2^{j^{\prime}}$. The unique solution of the last equation is $\left(y, j^{\prime}\right)=(1,2)$. Hence, $(3,1,8)$ is the unique solution.

Corollary 2.1. $(3,1,2)$ is a unique non-negative integer solution $(x, y, t)$ for the Diophantine equation $11+2^{x+2}+(7) 3^{y}=t^{6}$, where $x, y$ and $t$ are non-negative integers.

P r o of. Let $x, y$ and $t$ be non-negative integers such that $11+2^{x+2}+(7) 3^{y}=t^{6}$. Let $z=t^{3}$. Then $11+2^{x+2}+(7) 3^{y}=z^{2}$. By Theorem 2.1, we obtain that $(x, y, z)=(1,3,8)$. Then $t^{3}=z=8$. Hence, $t=2$.

\section{Open problem}

Let $a, b$ and $c$ be positive odd prime numbers such that $(a, b, c)=1$. We may ask for the set of all solutions $(x, y, z)$ for the Diophantine equation

$$
a+b 2^{x}+c 3^{y}=z^{2}
$$

where $x, y$ and $z$ are non-negative integers.

Acknowledgement. We would like to thank the editor and the referee for the opportunity to revise our manuscript. 


\section{ON THE DIOPHANTINE EQUATION $11+2^{x+2}+(7) 3^{y}=z^{2}$}

\section{REFERENCES}

[1] SUVARnAmani, A.: Solutions of the Diophantine equation $2^{x}+p^{y}=z^{2}$, Int. J. Math. Sci. Appl. 1 (2011), 1415-1419.

[2] CATAlAn, E.: Note extraite d'une lettre adressée a l'éditeur, J. Reine Angew. Math. 27 (1844), p. 192.

[3] MIHAILESCU, P.: Primary cycolotomic units and a proof of Catalan's conjecture, J. Reine Angew. Math. 27 (2004), 167-195.

[4] CHOtChaisthit, S.: On the Diophantine equation $4^{x}+p^{y}=z^{2}$ where $p$ is a prime number, Amer. J. Math. Sci. 1 (2012), 191-193.

[5] YU, Y.-LI, X.: The exponential Diophantine equation $2^{x}+b^{y}=z^{c}$, Sci. World J. 2014 (2014), 1-3. http://dx.doi.org/10.1155/2014/401816

Received February 20, 2017
Department of Mathematics University of Mouloud Mammeri 15000 Tizi-Ouzou ALGERIA

E-mail: sadani.idir@yahoo.fr 